\section{The cost of treating mucormycosis with isavuconazole compared with standard therapy in the UK}

\author{
Emma Bagshaw', Daniel Kuessner ${ }^{*, 2}$, Jan Posthumus², Cesar Escrig ${ }^{3}$, Michael \\ Blackney', Sebastian Marcel Heimann ${ }^{4} \&$ Oliver Andreas Cornely ${ }^{4,5}$
}

\begin{abstract}
Aim: Mucormycosis is a fungal infection associated with high mortality. Until recently, the only licensed treatments were amphotericin B (AMB) formulations. Isavuconazole (ISAV) is a new mucormycosis treatment. A UK-based economic model explored treatment costs with ISAV versus liposomal AMB followed by posaconazole. Materials \& methods: As a matched casecontrol analysis showed similar efficacy for ISAV and AMB, a cost-minimization approach was taken. Direct costs - drug acquisition, monitoring and administration, and hospitalization costs - were estimated from the National Health Service perspective. Results: Per-patient costs for ISAV and liposomal AMB + posaconazole were UK£26,810 and UK£41,855, respectively, with savings primarily driven by drug acquisition and hospitalization costs. Conclusion: ISAV may reduce costs compared with standard mucormycosis therapy.
\end{abstract}

First draft submitted: 19 December 2016; Accepted for publication: 24 January 2017;

Published online: 13 February 2017

Mucormycosis is a rapidly progressive and life-threatening invasive fungal disease [1,2]. Although rare - affecting around 0.06-0.16 people per 10,000 globally each year [3-5] - the threat of mucormycosis is growing, as the size of the at-risk population, comprising mainly immunocompromised and critically ill patients, is increasing [6]. Mortality rates for mucormycosis of $17-66 \%$ have been reported in recent studies $[1-2,6-10]$; however, these can rise to over $90 \%$ in cases of disseminated infection [11].

The cost of treating patients with mucormycosis is considerable, with total costs more than threefold higher for mucormycosis patients compared with matched controls at high risk of mucormycosis infection [7]. The contribution of antifungal drugs toward total treatment costs is substantial [12,13]; one evaluation reported antifungal costs of UK£17,752 for hematology patients with proven/probable invasive fungal infections in 2010 [12]. Surgical debridement and adverse events (AEs) management can also add to total treatment costs and increase the duration of hospital stays [14,15].

Until recently, the only licensed antifungal therapies for mucormycosis were amphotericin B (AMB) formulations, with liposomal AMB (L-AMB) currently the standard of care in Europe [14]. However, L-AMB carries a considerable risk of renal toxicity $[16,17]$, which has been linked with dose reductions and discontinuations $[17,18]$. It is also not available as an oral formulation [16] and,

\section{KEYWORDS}

- cost • economic

- healthcare $\bullet$ invasive

fungal infection

- isavuconazole

- liposomal amphotericin B

- mucormycosis

- posaconazole 
if outpatient maintenance therapy is required, an off-label step-down to oral posaconazole (POSA) has been suggested [19]. However, there is no evidence from prospective clinical trials to support the use of POSA in mucormycosis [20], and switches in drug class, such as this, carry the risk of treatment failure or disease progression.

Isavuconazole (ISAV) is a broad-spectrum, triazole antifungal [21], indicated in the EU in adults for the treatment of mucormycosis in patients for whom AMB is inappropriate, and for invasive aspergillosis [22]. ISAV's efficacy in the primary treatment of mucormycosis was comparable with that of $\mathrm{AMB} \pm \mathrm{POSA}$ in a matched case-control analysis of ISAV-treated patients with proven/probable mucormycosis from the global, Phase III, single-arm VITAL study with AMB (mainly L-AMB) \pm POSAtreated patients from the FungiScope ${ }^{\mathrm{TM}}$ global registry database of emerging fungal infections (day 42 crude all-cause mortality $33 \%$ of 21 ISAV patients vs 39\% of 33 AMB patients) [23]. ISAV has also been shown to be well tolerated compared with voriconazole, the standard of care therapy for invasive aspergillosis [24].

To evaluate the impact of ISAV with respect to healthcare resource use, a health-economic model was developed to estimate the per-patient cost of treating mucormycosis in the UK with ISAV alone compared with L-AMB followed by maintenance POSA.

\section{Materials \& methods}

\section{- Model structure \& key assumptions}

A cost-minimization approach was chosen to model the per-patient cost of mucormycosis treatment with ISAV compared with a regimen of L-AMB followed by maintenance therapy with POSA. As the matched case-control analysis of the VITAL study and FungiScope registry demonstrated no significant difference in day 42 all-cause mortality between ISAV and AMB \pm POSA in the primary treatment of mucormycosis [23], ISAV and L-AMB + POSA were assumed to have equivalent efficacy and only differences in costs between the treatments were considered in the model. Treatment modalities for ISAV and $\mathrm{L}$-AMB were assumed to be as per the regimens described in the matched case-control analysis.

The model was developed from the perspective of the UK National Health Service, and evaluated direct treatment costs incurred by treating patients with mucormycosis. Total costs comprised drug acquisition, intravenous (iv.) drug preparation and administration, monitoring, and hospitalization costs (Figure 1).

The model time horizon was from onset to completion of mucormycosis treatment, at either resolution of the Mucorales infection or death. Costs were calculated in $2016 \mathrm{UK} £$, with source values adjusted using a UK consumer price index where necessary [25].

\section{- Model parameters}

\section{Drug acquisition}

Drug acquisition costs included only the cost of ISAV, L-AMB and POSA. Dosing for ISAV was calculated in accordance with its Summary of Product Characteristics (SmPC; $2 \times 100 \mathrm{mg}$ oral capsules per day or $1 \times 200 \mathrm{mg}$ vial per day, loading dose on day 1 and 2 of $600 \mathrm{mg}[3 \times 200$ $\mathrm{mg}$ ]) [22]. As POSA is not indicated for mucormycosis, the doses recommended in its SmPC for the treatment of refractory invasive fungal infections [26] were used in the model $(3 \times 100$ mg oral tablets per day, loading dose on day 1 of $600 \mathrm{mg}$ [ $2 \times 300 \mathrm{mg}])$. The model considered the costs of the tablet form and not the oral suspension (which was received by the FungiScope cohort), as this is now recommended owing to higher bioavailability [21,22]. For L-AMB, a daily dose of $5 \mathrm{mg} / \mathrm{kg}$ was assumed, based on the median dose of L-AMB received by the FungiScope cohort [23] and as recommended in European clinical guidelines [14]. L-AMB dosing requirements were calculated using the patient weight distribution from the mucormycosis primary therapy population of the VITAL study (mean $75.9 \mathrm{~kg}$, SD $19.9 \mathrm{~kg}$ ).

In the VITAL study, $14.3 \%$ of mucormycosis patients who received ISAV as primary therapy were initiated on oral therapy; this proportion was therefore also assumed in the model. Treatment durations for ISAV (15.5 days iv. therapy followed by 133.5 days oral therapy; patients initiated on oral therapy received 0 days iv. and 149.0 days oral therapy) and L-AMB (27.2 days iv. therapy) were derived from the means of the mucormycosis primary therapy population of the VITAL study and the AMB-treated cohort of the FungiScope registry, respectively. As too few FungiScope patients received POSA to ensure an adequate sample size for the treatment duration ( $\mathrm{n}=$ 12), a conservative assumption was made that the duration of POSA would be set to equal the mean total duration of ISAV treatment 


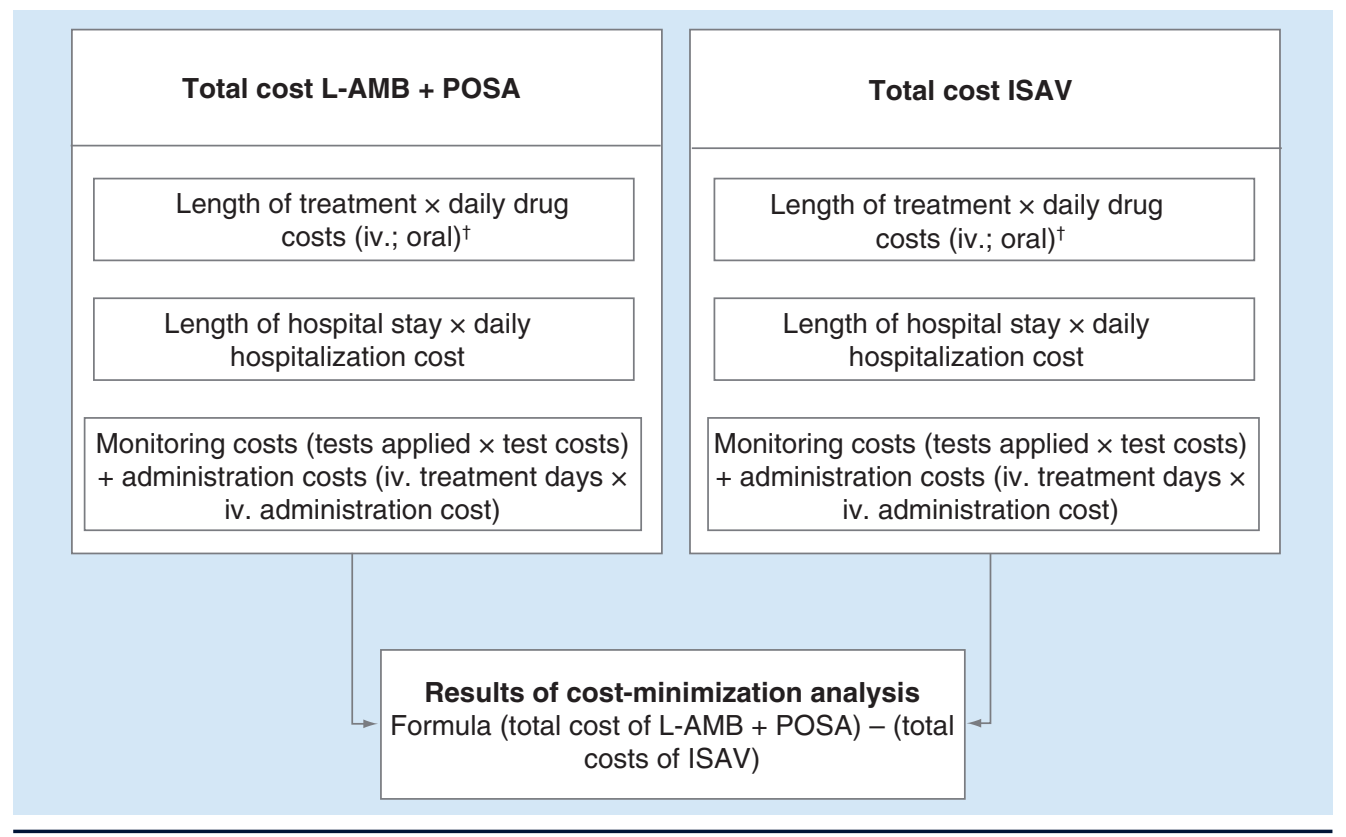

Figure 1. Model structure.

'Including loading dose as applicable.

ISAV: Isavuconazole; iv.: Intravenous; L-AMB: Liposomal amphotericin B; POSA: Posaconazole.

in the VITAL study minus the mean duration of iv. AMB treatment in the FungiScope cohort. Drug acquisition costs for L-AMB and POSA were taken from the British National Formulary [27], while the unit cost of ISAV was taken from the Monthly Index of Medical Specialities [28] (Table 1).

\section{Monitoring \& administration}

The costs of performing urinalysis, serum creatinine tests and liver-function tests were included in the model (Table 1). Based on the AEs and precautions listed in each product's SmPC, it was assumed that patients treated with L-AMB + POSA would require weekly monitoring with all three tests and patients treated with ISAV would have fortnightly liver-function testing only.

Therapeutic drug monitoring (Table 1) was assumed to be required twice over the course of treatment for L-AMB + POSA, based on British Society for Medical Mycology recommendations to perform therapeutic drug monitoring in patients receiving POSA [33]. Therapeutic drug monitoring is not a standard recommendation for ISAV [22], and was therefore not included for the ISAV arm of the model.

iv. administration costs (Table 1) were based on an administration time of $5 \mathrm{~min}$ and $51 \mathrm{~s}$ published elsewhere [31], and nursing salaries as reported by the Personal Social Services Research Unit [32].

\section{Hospitalizations}

Hospitalization costs (Table 1) were estimated by multiplying the duration of hospital stay by the daily cost of a hematology ward bed [12], as hematology patients were considered to be representative of the patient group that is at risk of mucormycosis $[6,34]$. For ISAV, the length of hospital stay was set to 19.3 days - the mean duration of initial hospitalization of patients who received ISAV as primary therapy in VITAL. For L-AMB + POSA, patients were assumed to remain hospitalized for the duration of iv. therapy (27.2 days).

\section{- Scenario analyses}

Scenario analyses were performed to test uncertainty in the model parameters. The base-case analysis assumed that most ISAV patients started on iv. therapy. However, as both the iv. and oral formulations of ISAV can be given as initial therapy, and given the potentially large cost-impact if the proportion of patients on either formulation were to be changed, two scenario analyses were performed in which this composition was altered. In the first, $100 \%$ of ISAV patients started on oral therapy and in the second, $100 \%$ started on iv., before moving to oral therapy as appropriate. 


\begin{tabular}{|c|c|}
\hline Cost factor & Unit cost (£) \\
\hline \multicolumn{2}{|l|}{ Drugs: ${ }^{+}$} \\
\hline - ISAV (200 mg vial) & 297.84 \\
\hline - ISAV (14-pack of $100 \mathrm{mg}$ tablets) & 599.28 \\
\hline - L-AMB (50 mg vial) & 82.19 \\
\hline - POSA (24-pack of $100 \mathrm{mg}$ tablets) & 595.96 \\
\hline Urinalysis, serum creatinine test, liver function test ${ }^{\ddagger}$ & 0.83 \\
\hline Therapeutic drug monitoring ${ }^{\S}$ & 58.51 \\
\hline iv. administration" & 3.53 \\
\hline Hospital bed day" & 523.33 \\
\hline \multicolumn{2}{|c|}{$\begin{array}{l}\text { 'Based on British National Formulary [27] and Monthly Index of Medical Specialities list prices [28]. } \\
\text { "Based on Scottish health service costs for clinical chemistry tests [29]. } \\
\text { 'Based on the price listed by the Mycology Reference Centre, Manchester [30]. } \\
\text { "Based on an administration time of } 5 \text { min and } 51 \text { s published elsewhere [31], and nursing salaries as reported by the Personal Social } \\
\text { Services Research Unit [32]. } \\
\text { "Based on the daily cost of a hematology ward bed [12]. }\end{array}$} \\
\hline
\end{tabular}

A further scenario analysis was performed, in which the duration of L-AMB + POSA treatment was varied. The base-case analysis assumed an L-AMB duration equal to the mean duration of $A M B$ received in the FungiScope cohort. The duration of POSA was calculated by taking this from the mean duration of ISAV in the VITAL primary therapy population. In the scenario analysis, the L-AMB and POSA durations were set to the mean duration of $\mathrm{AMB}$ and POSA administered to the subset of FungiScope patients who received AMB followed by POSA $(\mathrm{n}=12 ; 25.3$ days L-AMB; 200.4 days POSA).

Finally, although the base-case analysis was undertaken in the primary treatment setting, ISAV is indicated when AMB is inappropriate. To estimate the cost of ISAV treatment in this indication, and to determine whether costs would change across patient populations, two additional analyses were performed. The first used the mean duration of therapy for VITAL patients with disease refractory to prior antifungal treatment $(\mathrm{n}=11,91 \%$ of whom received prior AMB; 22.8 days iv. therapy, 94.2 days oral therapy) and the second used the mean duration of therapy of patients intolerant to prior treatment $(n=5,60 \%$ of whom received prior $\mathrm{AMB}$; 17.3 days iv. therapy, 80.1 days oral therapy). The results of these analyses were compared with the cost of ISAV treatment in the primary setting. For these analyses, comparisons with L-AMB + POSA were not considered suitable, given L-AMB's primary-treatment indication.

\section{Results}

\section{- Base case}

In the base-case analysis, the total per-patient cost of mucormycosis treatment was UK£26,810 with ISAV versus UK£41,855 with L-AMB + POSA (Table 2 \& Figure 2). This represents a saving of $36 \%$ with ISAV relative to L-AMB + POSA. Savings were primarily driven by lower iv. drug acquisition costs for ISAV than for L-AMB (UK£4978 vs UK£18,189) in addition to lower hospitalization costs (UK£10,100 vs $U K £ 14,235)$. The combined cost of monitoring and administration was also lower with ISAV than with L-AMB + POSA (UK£64 vs UK£269).

\section{- Scenario analyses}

Several scenario analyses were performed. In the first two, the proportion of ISAV patients initiated on iv. or oral therapy was varied. When $100 \%$ of ISAV patients started on oral therapy, total per-patient costs were UK£18,592 less with ISAV than with L-AMB + POSA, and when $100 \%$ of ISAV patients started on iv. therapy, per-patient costs were $U K £ 14,813$ less than with L-AMB + POSA (Figure 3).

In another scenario analysis, the treatment durations for L-AMB + POSA were set to the means derived from the subset of FungiScope patients who received AMB + POSA. Here, ISAV was UK£19,633 less costly per patient than the comparator combination (Figure 4).

In the final scenario analyses, the duration of ISAV treatment was taken from that of VITAL study patients unsuitable for AMB treatment. 


\begin{tabular}{|c|c|c|c|c|c|}
\hline \multirow[t]{2}{*}{ Cost source } & \multicolumn{2}{|c|}{ ISAV } & \multicolumn{2}{|c|}{ L-AMB + POSA } & \multirow{2}{*}{$\begin{array}{l}\text { (L-AMB + POSA) - (ISAV) } \\
\text { Savings } £(\% \text { change) }\end{array}$} \\
\hline & Resource use & Cost (£) & Resource use & Cost (£) & \\
\hline iv. drug & 15.5 days $^{\dagger}$ & 4978 & 27.2 days & 18,189 & $13,210(73)$ \\
\hline Oral drug & 133.5 days $^{\dagger}$ & 11,668 & 121.8 days & 9163 & $-2504(-27)$ \\
\hline Total drug acquisition & 149.0 days & 16,646 & 149.0 days & 27,352 & $10,706(39)$ \\
\hline Hospitalization & 19.3 days & 10,100 & 27.2 days & 14,235 & $4134(29)$ \\
\hline Monitoring and administration & 11.6 tests $^{\ddagger}$ & 64 & 68.9 tests $^{\ddagger}$ & 269 & $204(76)$ \\
\hline Total costs & & 26,810 & & 41,855 & $15,045(36)$ \\
\hline \multicolumn{6}{|c|}{$\begin{array}{l}\text { †Patients initiated on oral therapy received } 0.0 \text { days iv. and } 149.0 \text { days oral therapy. } \\
\text { fISAV patients underwent } 11.6 \text { liver-function tests; L-AMB + POSA patients underwent } 2.0 \text { instances of therapeutic drug monitoring plus } 22.3 \text { of each of the following: urinalysis, } \\
\text { serum creatinine tests and liver-function tests. } \\
\text { ISAV: Isavuconazole; iv: Intravenous; L-AMB: Liposomal amphotericin B; POSA: Posaconazole. }\end{array}$} \\
\hline
\end{tabular}

When the treatment duration of patients with refractory disease was used, total per-patient costs for ISAV were UK£25,425, compared with $\mathrm{UK} £ 26,810$ in the base-case analysis (Figure 5). When the duration of ISAV treatment was taken from VITAL study patients intolerant to prior antifungal treatment, total per-patient costs for ISAV were UK£22,727.

\section{Discussion}

A health-economic model was developed to compare the per-patient costs of treating mucormycosis with ISAV compared with L-AMB followed by oral maintenance POSA in the UK. The model estimated that use of ISAV as therapy against mucormycosis is likely to lead to substantial cost savings relative to L-AMB + POSA, primarily attributable to lower drug acquisition costs for ISAV compared with L-AMB, as well as lower hospitalization costs for the ISAV regimen.

Several scenario analyses were performed. When the proportion of ISAV patients starting on iv. or oral therapy was varied, ISAV remained cost saving relative to L-AMB + POSA. Similarly, when the duration of L-AMB + POSA treatment was altered to reflect the duration of treatment in the subset of FungiScope patients who received AMB followed by POSA, ISAV remained cost saving. When the duration of ISAV treatment was changed to that observed with refractory or intolerant patients in the VITAL study, total treatment costs were

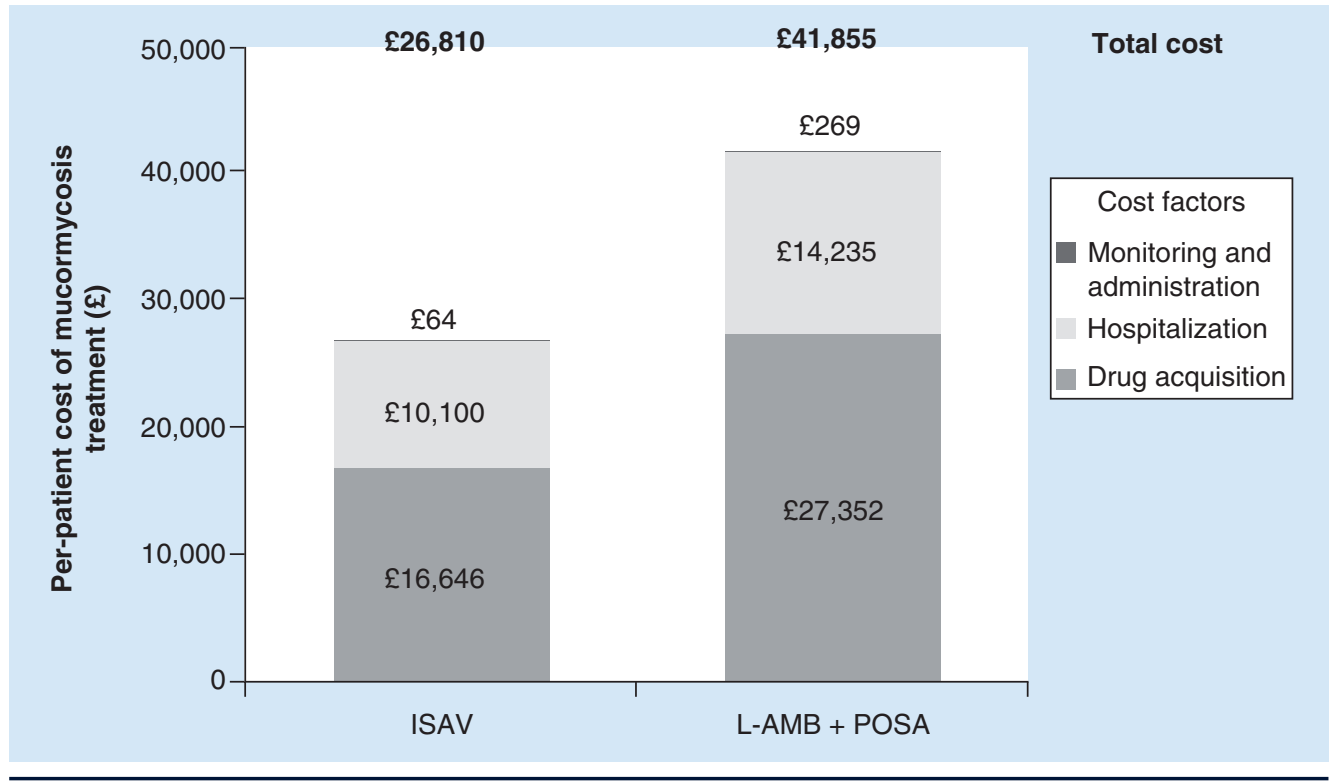

Figure 2. Results of base-case cost-minimization analysis.

ISAV: Isavuconazole; L-AMB: Liposomal amphotericin B; POSA: Posaconazole. 


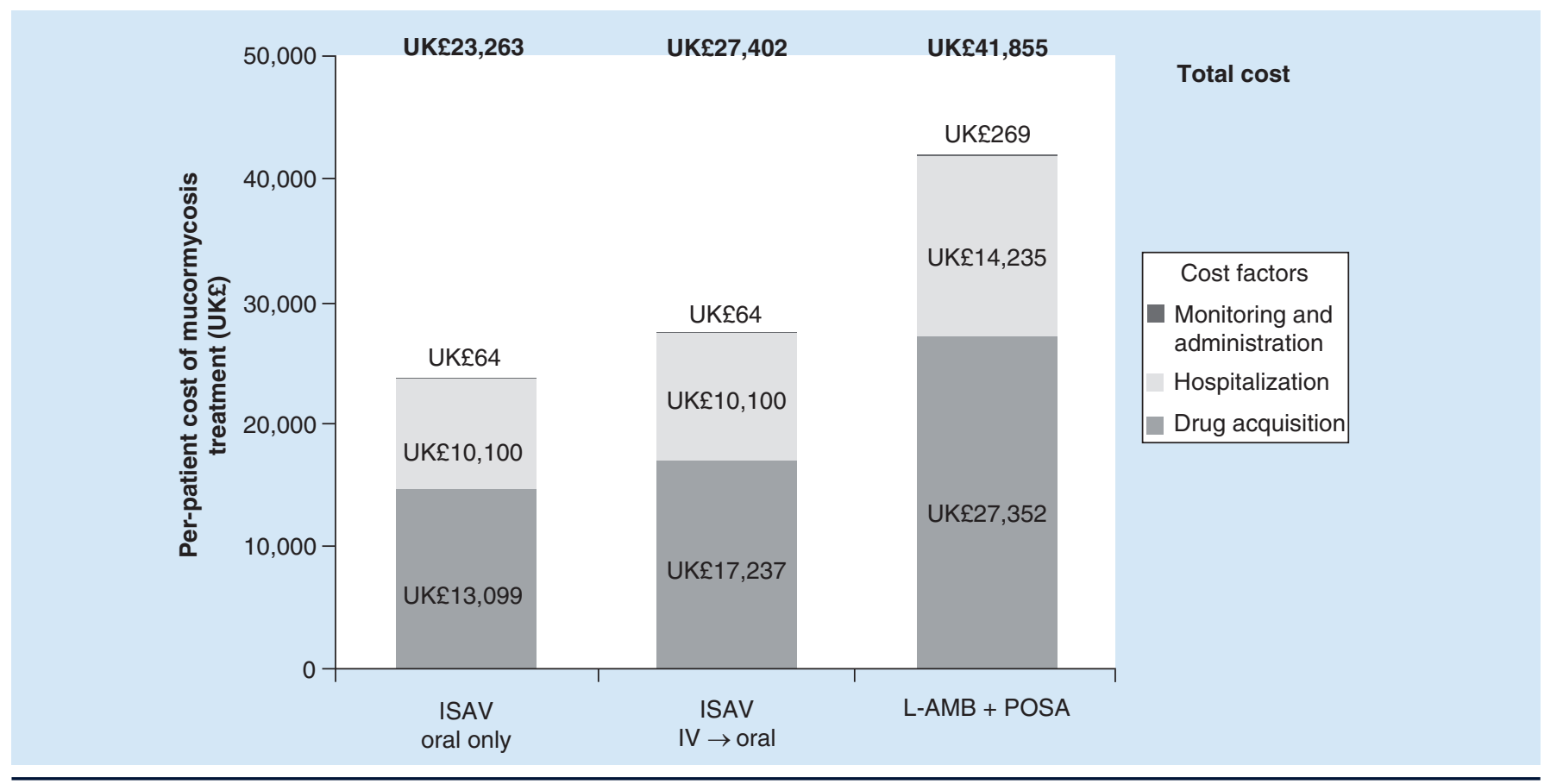

Figure 3. Results of scenario analyses: variation in proportion of isavuconazole patients starting on oral or iv. therapy. ISAV: Isavuconazole; iv.: Intravenous; L-AMB: Liposomal amphotericin B; POSA: Posaconazole.

similar to those for ISAV in the primary therapy setting, suggesting that ISAV treatment costs are likely to remain stable across different populations.

Given the rarity of mucormycosis, few studies have examined the cost of treatment; this is the first to evaluate the cost of mucormycosis treatment with ISAV. However, the key underlying assumption of the cost-minimization analysis - that of equal efficacy between ISAV and $\mathrm{AMB}$ - can be verified by comparison of outcomes in the VITAL study with those of historical studies of AMB-treated control populations. Three studies have been identified that can be considered similar in design to VITAL, as they included at least 20 patients treated with AMB-based regimens (majority L-AMB) and measured response to treatment according to accepted definitions from the European Organization for Research and Treatment of Cancer/Invasive Fungal Infections Cooperative Group and the National Institute of Allergy and Infectious Diseases Mycoses Study Group [35]. All of these studies reported similar rates of complete or partial overall response to $\mathrm{AMB}$ therapy at the end of treatment (ranging from 32 to $42 \%[8,18,36])$ to those seen with ISAV in the VITAL study, in both the overall VITAL mucormycosis population (31\%), and the subset receiving primary therapy (32\%) [23] (Table 3).
In the three historical control studies, the daily doses of AMB administered were generally higher than the median dose of L-AMB in the FungiScope cohort, which was used for the model (Lanternier et al. $10 \mathrm{mg} / \mathrm{kg}$ [18], Shoham et al. 3-14 mg/kg [36], Xhaard et al. not reported [8], FungiScope cohort $5 \mathrm{mg} / \mathrm{kg}$ [23]). Therefore, the modeled savings with ISAV relative to L-AMB + POSA described here may be considered conservative estimates.

A limited number of studies have investigated the cost of treating mucormycosis using AMB. Ibrahim et al. modeled data from a retrospective study to estimate the annual in-hospital cost of mucormycosis to the US healthcare system at US $\$ 97,743$. They found that the average drug costs associated with AMB formulations amounted to US $\$ 14,085$ (2007) - equivalent to $\sim \mathrm{UK} £ 7,201$ - per patient [13]. When compared with the cost of iv. L-AMB generated by our model (UK£18,189), this value seems low. However, the Ibrahim study analyzed the cost of various AMB formulations, whereas the model presented here considered only the recommended formulation, L-AMB [14]. In addition to drug costs, Ibrahim et al. found the average duration of hospitalization to be 35 days. This is greater than the length of stay for L-AMB used in the current model and suggests that estimates of 
hospitalization costs for L-AMB in the model may be conservative.

The model has several limitations. In the case of a rare disease like mucormycosis, where the availability of data is low, creating an accurate model is particularly challenging, and indirect evidence and assumptions must be used more frequently than is desirable. In addition, as the input values were based on a small number of patients (21 primary therapy patients from VITAL for ISAV and 33 from the FungiScope registry for L-AMB + POSA), the current model did not include sensitivity analyses; any results derived under these conditions would not be meaningful. A head-to-head trial between ISAV and L-AMB + POSA, reporting both clinical and resource use outcomes, would be preferable; however, the feasibility of conducting such a trial in a rare disease is low [37]. Future experience with ISAV in clinical practice will be useful to corroborate many of the assumptions made in the current model and will provide data with respect to cost-effectiveness in the real-world setting.

In addition to excluding the effect of AEs on patient utility, the current model did not evaluate their cost impact. The available AE data for ISAV and L-AMB were considered too different to make a robust comparison. Given that $\mathrm{AE}$ rates would have been sourced from separate trials and that the types of AEs commonly associated ISAV and L-AMB are very different in nature $[16,22]$, a standardized method of comparison would be very challenging to develop.

The model did not include several in-hospital costs likely to be incurred during mucormycosis treatment, namely the costs of surgery, imaging, or any additional treatments. For instance, nonmucormycosis-active antifungals, such as voriconazole or fluconazole, may be given prior to the modeled treatments, before confirmation of diagnosis and administration of targeted treatment. Although these costs could vary between ISAV and L-AMB + POSA, with a large potential impact on the total cost of mucormycosis treatment, they were not included in the model, as there is no evidence to suggest a difference in the need for surgery or prior treatment between ISAV and L-AMB. Had these costs been included, total costs would have likely risen by the same amount for each comparator. It should also be noted that use of ISAV or L-AMB early in the course of treatment may reduce costs associated with the inappropriate use of narrower-spectrum antifungals that lack activity in mucormycosis.

A further point is that cost-minimization modeling permits only a narrow scope of outcomes to be presented and limits the comparisons that can be made between treatments with respect to their economic impacts. Although,

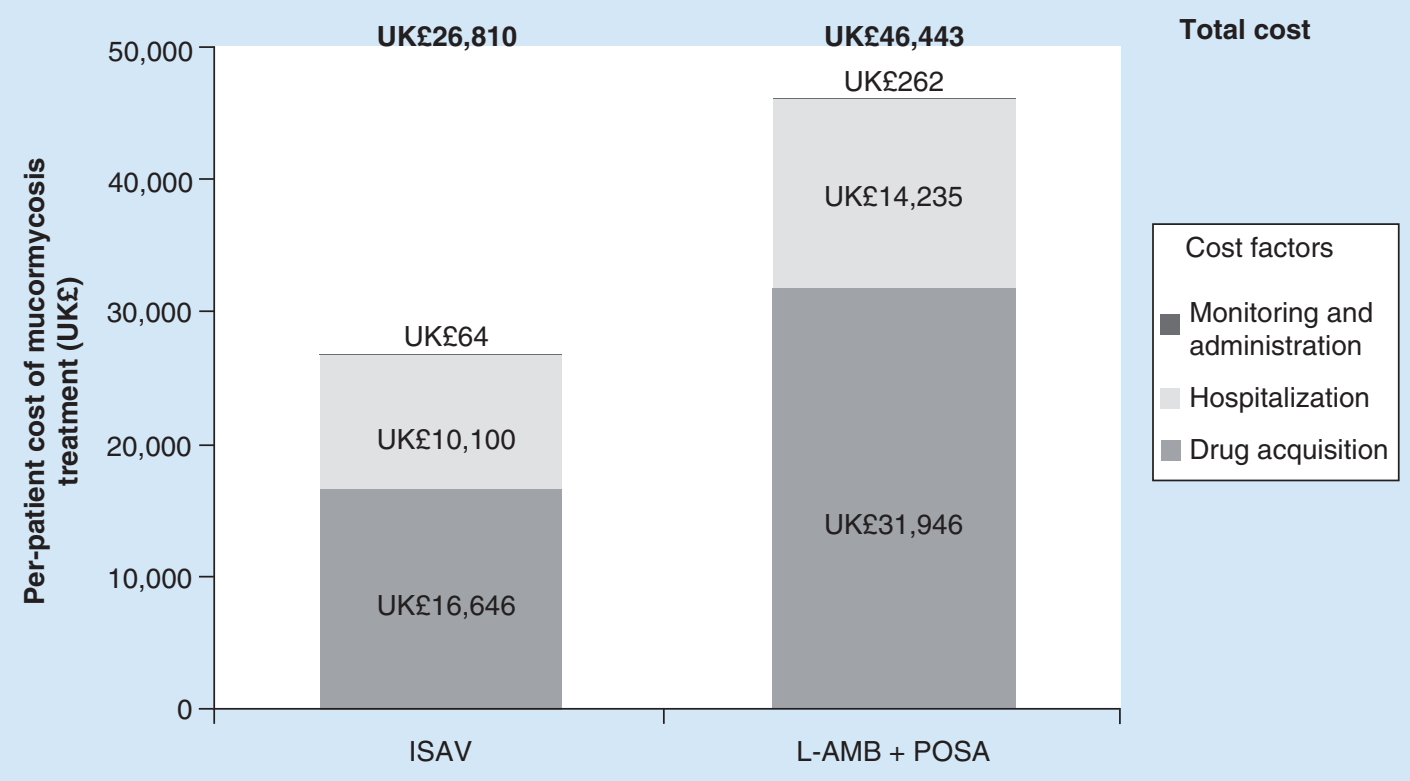

Figure 4. Results of scenario analysis: variation in duration of liposomal amphotericin B + posaconazole treatment based on FungiScope ${ }^{\mathrm{TM}}$ cohort.

ISAV: Isavuconazole; L-AMB: Liposomal amphotericin B; POSA: Posaconazole. 
RESEARCH ARTICLE Bagshaw, Kuessner, Posthumus et al.

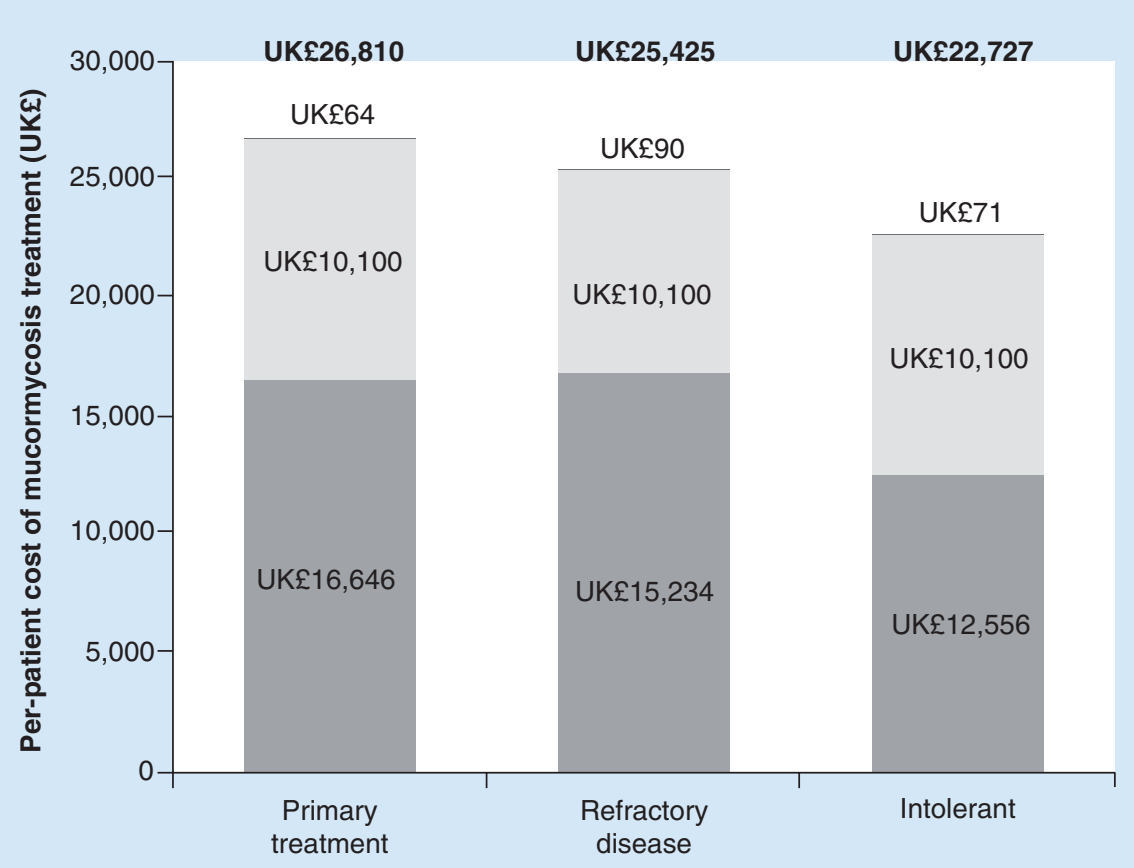

Total cost

\begin{tabular}{|l|}
\hline Cost factors \\
Monitoring and \\
administration \\
Hospitalization \\
Drug acquisition \\
\hline
\end{tabular}

Figure 5. Results of scenario analyses: variation in isavuconazole treatment duration based on primary, refractory and intolerant patients in VITAL.

ISAV: Isavuconazole.

for the current analysis, this approach was considered appropriate, a cost-utility approach, for example, would have allowed for a more rounded health-economic evaluation, including patient utility. As more data become available, more sophisticated analyses may be performed.

Finally, it should be noted that the applicability of our model is limited to the current UK perspective; any extrapolation of the results to other countries or time periods should be made with caution. Differences in healthcare systems, approaches to treating mucormycosis and changes in unit costs over time will affect treatment costs. Tied to this point, our analysis is limited by the fact that a global patient population was used to inform efficacy comparisons for the UK model. Efficacy outcomes and treatment regimens may have differed if a strictly UK-based population had been used, which could have affected resource use and costs.

\section{Conclusion}

Mucormycosis is a life-threatening fungal infection, with few treatment options. The

\begin{tabular}{|c|c|c|c|c|c|}
\hline \multirow{2}{*}{$\begin{array}{l}\text { Overall response to } \\
\text { treatment (\%) }\end{array}$} & \multicolumn{5}{|c|}{ Study population } \\
\hline & $\begin{array}{l}\text { Shoham et al. [36] } \\
n=28\end{array}$ & $\begin{array}{l}\text { Xhaard et al. }[8] \\
n=24\end{array}$ & $\begin{array}{l}\text { Lanternier et al. }[18] \\
n=33\end{array}$ & $\begin{array}{l}\text { VITAL, all mucormycosis } \\
\text { patients } n / n=35 / 37^{+}\end{array}$ & $\begin{array}{l}\text { VITAL, primary therapy } \\
\text { mucormycosis patients } n / n=19 / 21^{+}\end{array}$ \\
\hline Complete response & $\begin{array}{l}32 \text { complete }+ \\
\text { partial }\end{array}$ & 29 & 18 & 14 & 16 \\
\hline Partial response & & 13 & 18 & 17 & 16 \\
\hline Stable disease & 11 & $\begin{array}{l}58 \text { stable }+ \\
\text { progression }\end{array}$ & 12 & 29 & 32 \\
\hline Disease progression & 57 & & 52 & 40 & 37 \\
\hline
\end{tabular}


current standard of care, L-AMB, has been used for many years for the treatment of mucormycosis but has limitations, for example, it carries a risk of renal toxicity and is only available as an iv.-administered formulation. ISAV is a recently licensed treatment for mucormycosis in adults for whom AMB is inappropriate. ISAV has comparable efficacy to AMB, an acceptable safety profile, and is available in both iv. and oral formulations. Results from the current model suggest that the use of ISAV can potentially reduce the cost of treating patients with mucormycosis compared with a regimen of L-AMB followed by oral POSA. ISAV may prove to be a cost-effective option for the treatment of mucormycosis; however, further health-economic evaluations are needed to confirm this.

\section{Financial \& competing interests disclosure}

This study was supported by Basilea Pharmaceutica International Ltd, Basel. E Bagshaw and M Blackney are employees of Covance Market Access, which was commissioned by Basilea Pharmaceutica International Ltd to provide support with model development and the writing of this article. D Kuessner, J Posthumus and C Escrig are employees of Basilea Pharmaceutica International Ltd and hold stock options with Basilea Pharmaceutica Ltd. Within the past 36 months, $S$ Heimann has received research grants from the following organizations: Astellas,
Basilea, Gilead, Merck Sharp \& Dohme and, 3M. He has also received travel grants from Astellas, Merck Sharp \& Dohme, and Pfizer. He has acted as a speaker for Astellas and Merck Sharp \& Dohme, and as a consultant to Basilea. Within the past 36 months, O Cornely has received research grants from Actelion, Aranis, Astellas, AstraZeneca, Basilea, Bayer, Cidara, Duke University (NIH UM1AI104681), F2G, Gilead, GSK, Leeds University, MedPace, Melinta Therapeutics, Merck/MSD, Miltenyi, Pfizer, Rempex, Roche, Sanofi Pasteur, Scynexis, Seres Therapeutics and The Medicine Company. He has acted as a consultant to Anacor, Amplyx, Actelion, Astellas, Basilea, Cidara, Da Volterra, F2G, Gilead, Janssen Pharmaceuticals, Matinas, Menarini Ricerche, Merck/MSD, Paratek Pharmaceuticals, Scynexis, Seres, Summit, Vical and Vifor, and he has received lecture honoraria from Astellas, Basilea, Gilead and MerckI MSD. The authors have no other relevant affliations or financial involvement with any organization or entity with a financial interest in or financial conflict with the subject matter or materials discussed in the manuscript apart from those disclosed.

No writing assistance was utilized in the production of this manuscript.

\section{Open access}

This work is licensed under the AttributionNonCommercial-NoDerivatives 4.0 Unported License. To view a copy of this license, visit http://creativecommons.org/ licenses/by-nc-nd/4.0/

\section{EXECUTIVE SUMMARY}

- Mucormycosis is a rapidly progressive fungal infection, with a high risk of mortality, which affects mainly immunocompromised and critically ill people.

- The cost of treating patients with mucormycosis is substantial, largely owing to the prolonged use of high-cost antifungals.

- Liposomal amphotericin B (L-AMB) - the standard treatment for mucormycosis in the UK - carries a risk of renal toxicity and is only available as an intravenous-administered formulation.

- Isavuconazole (ISAV) is a new treatment for mucormycosis in adults for whom AMB is inappropriate.

- A cost-minimization model was developed from the perspective of the UK National Health Service to explore the perpatient cost (drug acquisition, monitoring and administration, and hospitalization costs) of treating mucormycosis with ISAV versus L-AMB followed by posaconazole (POSA).

- Total per-patient treatment costs were estimated to be UK£26,810 with ISAV versus UK£41,855 with L-AMB + POSA.

- Savings were primarily driven by lower drug acquisition costs with ISAV relative to L-AMB, alongside lower hospitalization costs, since the iv.-to-oral switch occurred earlier for ISAV patients than for those who received LAMB + POSA.

- ISAV consistently remained cost saving across various scenario analyses designed to test the model parameters with most uncertainty.

- ISAV appears to be a cost-effective option for the treatment of mucormycosis; however, further health-economic evaluations are warranted. 


\section{References}

Papers of special note have been highlighted as: - of interest; $\bullet$ of considerable interest

1 Chamilos G, Lewis RE, Kontoyiannis DP. Delaying amphotericin B-based frontline therapy significantly increases mortality among patients with hematologic malignancy who have zygomycosis. Clin. Infect. Dis. 47 , 503-509 (2008)

2 Skiada A, Pagano L, Groll AH et al. Zygomycosis in Europe: analysis of 230 cases accrued by the registry of the European Confederation of Medical Mycology (ECMM) Working Group on Zygomycosis between 2005 and 2007. Clin. Microbiol. Infect. 12, 1859-1867 (2011).

3 European Medicines Agency. Committee for Orphan Medicinal Products. Public summary of opinion on orphan designation. Isavuconazonium sulfate for the treatment of invasive aspergillosis (2014).

www.ema.europa.eu/docs/en_GB

4 Kontoyiannis DP, Yang H, Song J et al. Prevalence, clinical and economic burden of mucormycosis-related hospitalizations in the United States: a retrospective study. $B M C$ Infect. Dis. 16, 730 (2016).

5 Chakrabarti A, Sood P, Denning D. Estimating fungal infection burden in India using computational models: mucormycosis burden as a case study. Presented at: $23 \mathrm{rd}$ European Congress of Clinical Microbiology and Infectious Diseases. Berlin, Germany, 27-30 April 2013.

6 Bitar D, Lortholary O, Le Strat Y et al. Population-based analysis of invasive fungal infections, France, 2001-2010. Emerg. Infect. Dis. 20 (7), 1149-1155 (2014).

7 Zilberberg MD, Shorr AF, Huang H, Chaudhari P, Federico Paly V, Menzin J. Hospital days, hospitalization costs, and inpatient mortality among patients with mucormycosis: a retrospective analysis of US hospital discharge data. BMC Infect. Dis. 14, 310 (2014).

- Retrospective analysis of Healthcare Cost and Utilization Project - Nationwide Inpatient Sample, to determine inpatient mortality, length of hospital stay and hospitalization costs associated with mucormycosis in the USA.

8 Xhaard A, Lanternier F, Porcher R et al. Mucormycosis after allogeneic haematopoietic stem cell transplantation: a French multicentre cohort study (2003-2008). Clin. Microbiol. Infect. 18(10), E396-E400 (2012).

Lanternier F, Dannaoui E, Morizot G et al. A global analysis of mucormycosis in France: the RetroZygo study (2005-2007). Clin. Infect. Dis. 54(S1), S35-S43 (2012).

10 Vaezi A, Moazeni M, Rahimi MT, de Hoog $S$, Badali H. Mucormycosis in Iran: a systematic review. Mycoses 59(7), 402-415 (2016).

11 Petrikkos G, Skiada A, Lortholary O, Roilides E, Walsh TJ, Kontoyiannis DP. Epidemiology and clinical manifestations of invasive mucormycosis. Clin. Infect. Dis. 54(Suppl. 1), S23-S34 (2012).

- Review providing background information on mucormycosis, focusing on epidemiology, risk factors and clinical manifestations.

12 Mansour Ceesay M, Sadique Z, Harris R, Ehrlich A, Adams EJ, Pagliuca A. Prospective evaluation of the cost of diagnosis and treatment of invasive fungal disease in a cohort of adult haematology patients in the UK. J. Antimicrob. Chemother. 70, 1175-1181 (2015).

- Prospective study of hospital resource use by hematology patients at high risk of invasive fungal infection, to determine the direct cost of invasive fungal disease to the UK healthcare system.

13 Ibrahim AS, Edwards JE Jr, Bryant R, Spellberg B. Economic burden of mucormycosis in the Unites States: can a vaccine be cost-effective? Med. Mycol. 47, 592-600 (2009).

- Analysis of a model, based on a retrospective study, to estimate the annual cost to the US healthcare system of treating mucormycosis, with the aim of assessing the cost-efficacy of a potential vaccine for mucormycosis.

14 Cornely OA, Arikan-Akdagli S, Dannaoui E et al. ESCMID and ECMM joint clinical guidelines for the diagnosis and management of mucormycosis 2013. Clin. Microbiol. Infect. 20(Suppl. 3), 5-26 (2014).

- Current guidelines from the European Society for Clinical Microbiology and Infectious Diseases and the European Confederation of Medical Mycology for the diagnosis and treatment of mucormycosis.

15 Bates DW, Su L, Yu DT et al. Mortality and costs of acute renal failure associated with amphotericin B therapy. Clin. Infect. Dis. 32, 686-693 (2001).

16 AmBisome $50 \mathrm{mg}$ powder for solution for infusion (liposomal amphotericin B). Summary of product characteristics. Gilead Sciences Ltd. (2015). www.medicines.org.uk/emc/medicine/1236.

17 Cornely OA, Maertens J, Bresnik M et al. Liposomal amphotericin B as initial therapy for invasive mold infection: a randomized trial comparing a high-loading dose regimen with standard dosing (AmBiLoad Trial). Clin. Infect. Dis. 44, 1289-1297 (2007).

18 Lanternier F, Poiree S, Elie C et al. Prospective pilot study of high-dose $(10 \mathrm{mg} / \mathrm{kg} /$ day) liposomal amphotericin B (L-AMB) for the initial treatment of mucormycosis. J. Antimicrob. Chemother. 70 (11), 3116-3123 (2015).

19 Tacke D, Keohler P, Markiefka B, Cornely OA. Our 2014 approach to mucormycosis. Mycoses 57, 519-524 (2014).

20 Vehreschild JJ, Birtel A, Vehreschild MJ et al. Mucormycosis treated with posaconazole: review of 96 case reports. Crit. Rev. Microbiol. 39(3), 310-324 (2013).

21 Kovanda LL, Maher R, Hope WW. Isavuconazonium sulfate: a new agent for the treatment of invasive aspergillosis and invasive mucormycosis. Expert Rev. Clin. Pharmacol. 9(7), 887-897 (2016).

22 Basilea Pharmaceutica International Ltd. Cresemba $200 \mathrm{mg}$ powder for concentrate for solution for infusion. Summary of product characteristics. (2016). www.medicines.org.uk/emc/medicine/31236.

23 Marty FM, Ostrosky-Zeichner L, Cornely $\mathrm{OA}$ et al. Isavuconazole treatment for mucormycosis: a single-arm open-label trial and case-control analysis. Lancet Infect. Dis. 16(7), 828-837 (2016).

- Phase III VITAL study and matched case-control analysis of isavuconazole in mucormycosis.

24 Maertens JA, Raad II, Patterson TF et al. Isavuconazole versus voriconazole for primary treatment of invasive mould disease caused by Aspergillus and other filamentous fungi (SECURE): a Phase III, randomisedcontrolled, non-inferiority trial. Lancet 387(10020), 760-769 (2016).

25 Inflation.eu. Inflation Great Britain (2016). http://inflation.eu/inflation-rates/great

26 Noxafil $100 \mathrm{mg}$ gastro-resistant tablets (posaconazole). Summary of product characteristics. Merck Sharp \& Dohme Ltd. (2015). www.medicines.org.uk/emc/medicine/28880

27 National Institute for Health and Care Excellence. British National Formulary (2016). www.evidence.nhs.uk/formulary/bnf/current

28 Monthly Index of Medical Specialities. Cresemba (2016). www.mims.co.uk/drugs/infections-and

29 Information Services Division Scotland. Scottish Health Service Costs. R130x 
Laboratory Services 2014-15 (2015). http://www.isdscotland.org/Health-Topics

30 Mycology Reference Centre Manchester. Antifungal assays.

http://www.mycologymanchester.org

31 van Zanten ARH, Engelfriet PM, van Dillen K, van Keen M, Nuijten MJC, Polderman $\mathrm{KH}$. Importance of nondrug costs of intravenous antibiotic therapy. Crit. Care 7(6), R184-R190 (2003).

32 Personal Social Services Research Unit. Unit Costs of Health and Social Care 2015 (2015). www.pssru.ac.uk/project-pages/unit-costs/

33 Ashbee HR, Barnes RA, Johnson EM, Richardson MD, Gorton R, Hope WW.
Therapeutic drug monitoring (TDM) of antifungal agents: guidelines from the British Society for Medical Mycology. J. Antimicrob. Chemother. 69(5), 1162-1176 (2014).

34 Ceesay MM, Desai SR, Cleverley J et al. A comprehensive diagnostic approach using galactomannan, targeted $\beta$-D-glucan, baseline computerized tomography and biopsy yields a significant burden of invasive fungal disease in at risk haematology patients. Br. J. Haematol. 168(2), 219-229 (2015).

35 De Pauw B, Walsh TJ, Stevens DA et al. Revised definitions of invasive fungal disease from the European Organization for Research and Treatment of Cancer/Invasive Fungal
Infections Cooperative Group and the National Institute of Allergy and Infectious Diseases Mycoses Study Group (EORTC/ MSG) consensus group. Clin. Infect. Dis. 46(12), 1813-1821 (2008).

36 Shoham S, Magill SS, Merz WG et al. Primary treatment of zygomycosis with liposomal amphotericin B: analysis of 28 cases. Med. Mycol. 48(10), 511-517 (2010).

37 Cornely OA, Maher RM, Marty FM, Lee M, Vehreschild JJ, Vehreschild MJGT. The VITAL study: case control studies are hypothesis generating - author's reply. Lancet Infect. Dis. 16(8), 886-887 (2016). 